\title{
Predicting Modeling Method of Ship Radiated Noise Based on Genetic Algorithm
}

\author{
Guohui Li and Hong Yang \\ School of Electronic Engineering, Xian University of Posts and Telecommunications, Xi'an, Shaanxi 710121, China \\ Correspondence should be addressed to Guohui Li; lghcd@163.com
}

Received 10 March 2016; Revised 20 May 2016; Accepted 29 May 2016

Academic Editor: Yaguo Lei

Copyright (C) 2016 G. Li and H. Yang. This is an open access article distributed under the Creative Commons Attribution License, which permits unrestricted use, distribution, and reproduction in any medium, provided the original work is properly cited.

\begin{abstract}
Because the forming mechanism of underwater acoustic signal is complex, it is difficult to establish the accurate predicting model. In this paper, we propose a nonlinear predicting modeling method of ship radiated noise based on genetic algorithm. Three types of ship radiated noise are taken as real underwater acoustic signal. First of all, a basic model framework is chosen. Secondly, each possible model is done with genetic coding. Thirdly, model evaluation standard is established. Fourthly, the operation of genetic algorithm such as crossover, reproduction, and mutation is designed. Finally, a prediction model of real underwater acoustic signal is established by genetic algorithm. By calculating the root mean square error and signal error ratio of underwater acoustic signal predicting model, the satisfactory results are obtained. The results show that the proposed method can establish the accurate predicting model with high prediction accuracy and may play an important role in the further processing of underwater acoustic signal such as noise reduction and feature extraction and classification.
\end{abstract}

\section{Introduction}

Underwater acoustic signal processing is a major branch of signal processing. As road categories of underwater acoustic signal, ship radiated noise is generated by the nonlinearity of the marine environment and hull structure together, which contains a large amount of ship target's position, distance, and depth information [1]. The complex forming mechanism of underwater acoustic signal makes it difficult to establish the accurate predicting model. The modeling of underwater acoustic signal is the foundation of the underwater acoustic signal processing. Research shows that underwater acoustic signal not only has the characteristics such as being nonlinear, non-Gaussian, and nonstationary, but also has the characteristics of being chaos, fractal, and so on [2-5]. In such cases, it is possible to model the behaviour of the system and get short-term prediction of the system evolution. The potential usefulness of this approach is enormous because a wide variety of physical systems show a chaotic nature. A physical example of the above-mentioned situation is given by the physical nature of ship radiated noise.

Modeling effect has a direct impact on the result of subsequent processing. In order to establish the model of underwater acoustic signal effectively, some modeling methods are proposed. Modeling and prediction of underwater acoustic signal based on PSO and RBF neural network [6] are proposed. Experimental results show this algorithm has better performance in terms of training accuracy and convergence rate and also supports the modeling, prediction, and dynamic analysis of underwater acoustic signals. Prediction of underwater acoustic signals based on neural network [7] is proposed. Predictions of both simulated data and real ship radiated noise data are made using $\mathrm{BP}$ and RBF network. But those prediction methods do not give an accurate mathematical expression of underwater acoustic signal. In recent years, genetic algorithm is widely used in signal modeling. Fuel flow-rate modelling of transport aircraft for the climb flight using genetic algorithms is proposed [8]. Predicting underwater ocean noise with genetic algorithms is proposed [9]. There are few reports on the modeling of ship radiated noise using genetic algorithm. In view of this, this paper tries to propose a predicting modeling method of ship radiated noise based on genetic algorithm.

\section{Predicting Modeling Method of Ship Radiated Noise Based on Genetic Algorithm}

Studies have found that some seemingly similar random signals can be described by the deterministic nonlinear 
dynamic system [10]. Volterra series model is the most basic model of nonlinear dynamic system [11]. In theory, any nonlinear dynamical system can be characterized by Volterra series model. In particular, when the Volterra series model is used to represent a nonlinear dynamical system, it not only has clear physical meaning, but also has strong universality. Research shows that ship radiated noise has a definite nonlinear dynamic characteristic [2-5]. This conclusion provides a theoretical basis for the nonlinear dynamic modeling of underwater acoustic signal and provides a new approach for the prediction of underwater acoustic signal. The genetic algorithm has already shown itself to be robust approaches to determining the functional form. In order to get an accurate mathematical expression of underwater acoustic signal, the modeling method of ship radiated noise with genetic algorithm is proposed. First of all, a basic model framework is chosen. Secondly, each possible model is done with genetic coding. Thirdly, model evaluation standard is established. Fourthly, the operation of genetic algorithm such as crossover, reproduction, and mutation is designed. Finally, a prediction model of real underwater acoustic signal is established by genetic algorithm. Detailed procedure is as follows.

2.1. Selection of Basic Model. The Volterra series model is given by

$$
\begin{aligned}
y= & f\left(x_{1}, x_{2}, \ldots, x_{N}\right) \\
= & w_{0}+\sum_{i=1}^{N} w_{i} x_{i}+\sum_{i_{1}, i_{2}=1}^{N} w_{i_{1} i_{2}} x_{i_{1}} x_{i_{2}}+\cdots \\
& +\sum_{i_{1}, i_{2}, \ldots, i_{M}=1}^{N} w_{i_{1} i_{2} \cdots i_{M}} x_{i_{1}} x_{i_{2}} \cdots x_{i_{M}},
\end{aligned}
$$

where $M$ is Volterra model order and $N$ is variable number. A total item in (1) is $1+N+N^{2}+\cdots+N^{M}$. In these items, not everyone can reflect the internal rule of time series. Too much model items will decline the model prediction performance. So a new model is formed by selecting some items based on the Volterra model. The selection of model item is accomplished by genetic algorithm.

2.2. Genetic Code. In order to solve the model by genetic algorithm, the right items are chosen as the components of the model, and each item is needed to code.

The model item can have the general representation defined by

$$
\prod_{i=1}^{M} x_{i}^{n_{i}} \quad n_{i} \geq 0
$$

where $\sum_{i=1}^{N} n_{i} \leq M$

Equation (2) can be represented by a vector $a=$ $\left(n_{1}, n_{2}, \ldots, n_{m}\right)^{T}$. Such vectors are composed of a matrix which means a model. Each column vector of the matrix represents an item of the model, and the column number of the matrix is the item number of the model.
2.3. Model Evaluation Criteria. In the process of establishing the prediction model of the measured underwater acoustic signal, the prediction accuracy can be used as the evaluation criteria of the model. In this model, the model coefficients are calculated by the least square method. So the prediction precision of the model is increased with the model item. But actually increased polynomial does not necessarily reflect the inherent laws of the data. In order to solve this problem, a part of data is selected as the training data, and the rest is used as the evaluation data. For a certain model, the model coefficients are calculated by the training data and the least square method. The prediction error of the model which is calculated by the evaluation data is used as the evaluation criterion of the model.

2.4. Genetic Operation. Cross breeding is as follows. These items of the two models are sorted, respectively, according to certain rules. In two sequences, a crossover point is randomly selected; then the second half of the sequence is exchanged, so that two new models are obtained, which are the offspring of cross breeding.

Genetic variation is as follows. Firstly, one item of the model is randomly generated. Secondly, the original one of the model is randomly replaced. Lastly, genetic variation is completed.

2.5. Establishing Prediction Model. Combined with the optimal individual encoding and these coefficients, the mathematical expression of underwater acoustic signal is established.

\section{Modeling of Measured Underwater Acoustic Signal}

In the South China Sea, three types of ship radiated noise are measured in the same sea condition. They are, respectively, the first type of ships, the second type of ships, and the third type of ships, as shown in Figure 1. The sampling frequency of the ship signals is $20 \mathrm{kHz}$. The sample length of the data is 2048, and there are 50 samples for each type of ship data.

In the prediction, 6 points are used, the time interval between each point is 1 , the population size is 60 , the reproduction algebra is 20 , and the alienation probability is 0.03 .

The modeling process and results of three types of measured underwater acoustic signal are given, respectively, as follows.

3.1. Modeling of the First Type of Underwater Acoustic Signal. Optimal individual encoding is given by

$$
\left[\begin{array}{lllllll}
1 & 0 & 0 & 0 & 1 & 0 & 0 \\
0 & 1 & 0 & 0 & 1 & 0 & 1 \\
0 & 0 & 1 & 0 & 0 & 1 & 1 \\
0 & 0 & 0 & 1 & 0 & 2 & 0 \\
0 & 0 & 0 & 0 & 1 & 0 & 1
\end{array}\right] .
$$




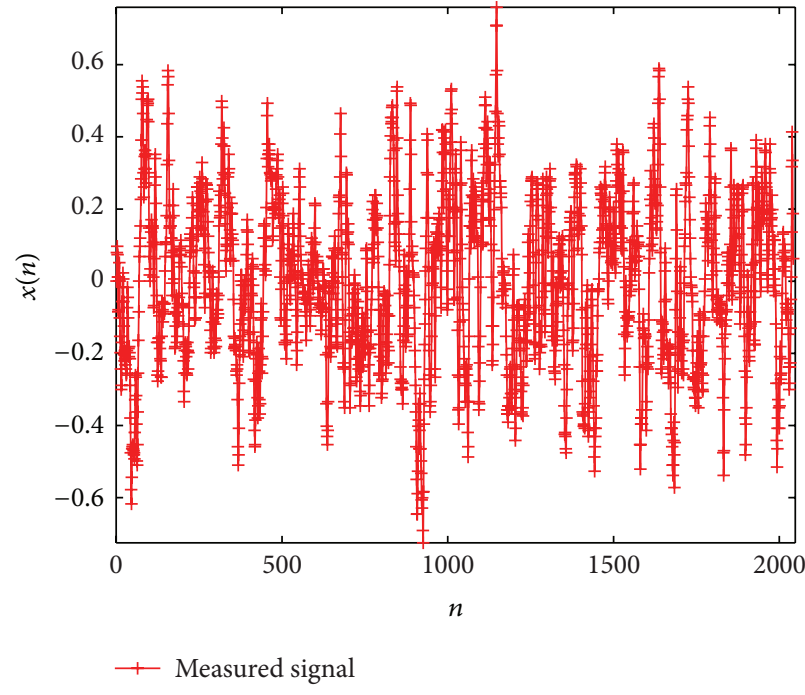

(a) Measured signal of the first type

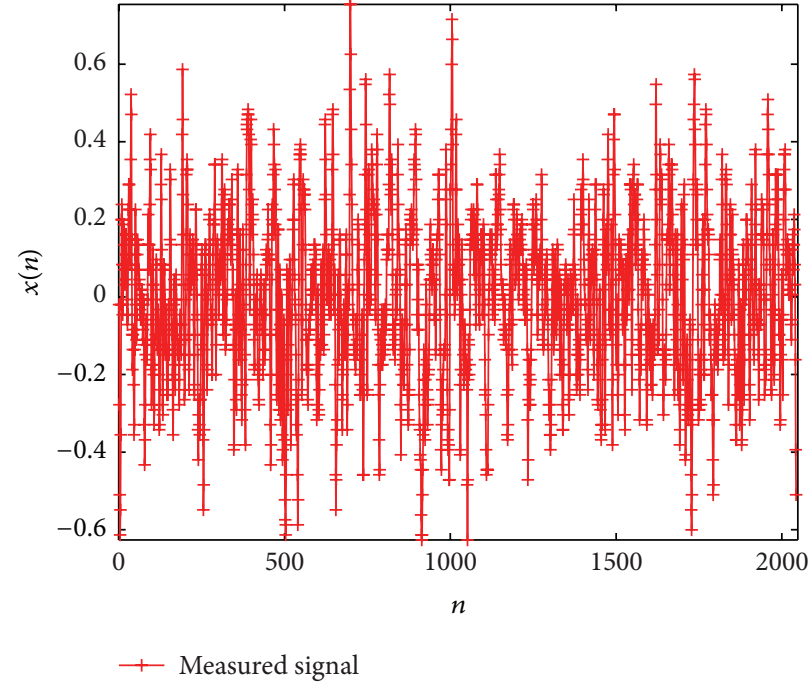

(b) Measured signal of the second type

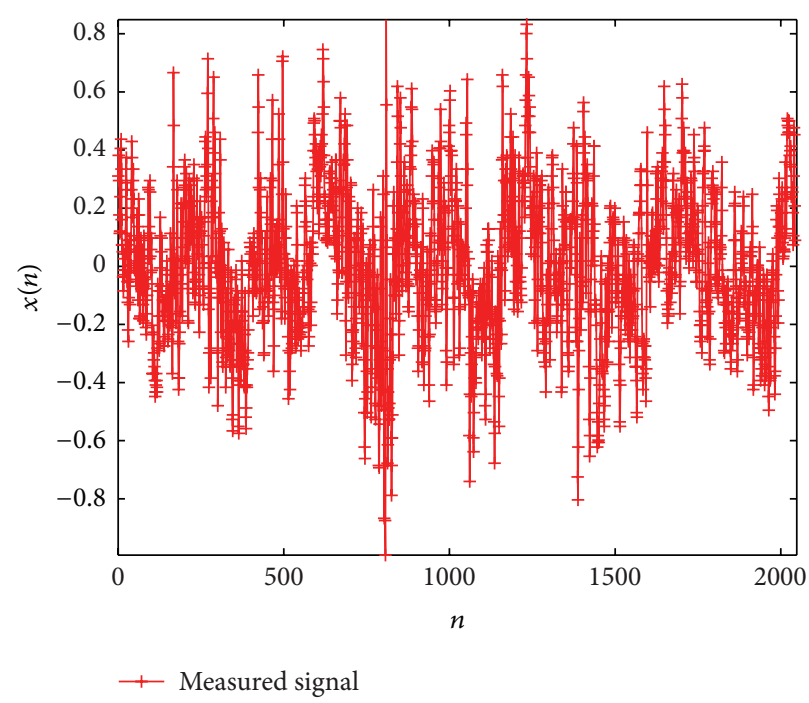

(c) Measured signal of the third type

FIGURE 1: Measured signal for the three types of ship radiated noise (2048 points).

The first line to the fifth line represent, respectively, $x(n-$ $1), x(n-2), x(n-3), x(n-4)$, and $x(n-5)$. The first column to the seventh column represent that the mathematical expression has seven components after modeling. The numerical value is 1 for its own, and the value is 2 for the square of its own. For example, the sixth column is $\begin{array}{llllllllll}0 & 0 & 1 & 2 & 0\end{array}$. That is, the sixth item of the mathematical expression after modeling is $x(n-3) x(n-4)^{2}$.

These coefficients obtained by the least squares method are $2.1129,-1.9148,1.0187,-0.2583,-0.6330,-0.5970$, and 1.2200 .

Combined with the optimal individual encoding and these coefficients, the mathematical expression of the first type of underwater acoustic signal is given by the nonlinear functional relation

$$
\begin{aligned}
x(n)= & 2.1129 x(n-1)-1.9148 x(n-2) \\
& +1.0187 x(n-3)-0.2583 x(n-4) \\
& -0.633 x(n-1) x(n-2) x(n-5) \\
& -0.597 x(n-3) x(n-4)^{2} \\
& +1.22 x(n-2) x(n-3) x(n-5),
\end{aligned}
$$

where $x(n)$ is the signal amplitude.

Measured signal and predicted signal for the first type of real ship radiated noise are shown in Figure 2. To facilitate 


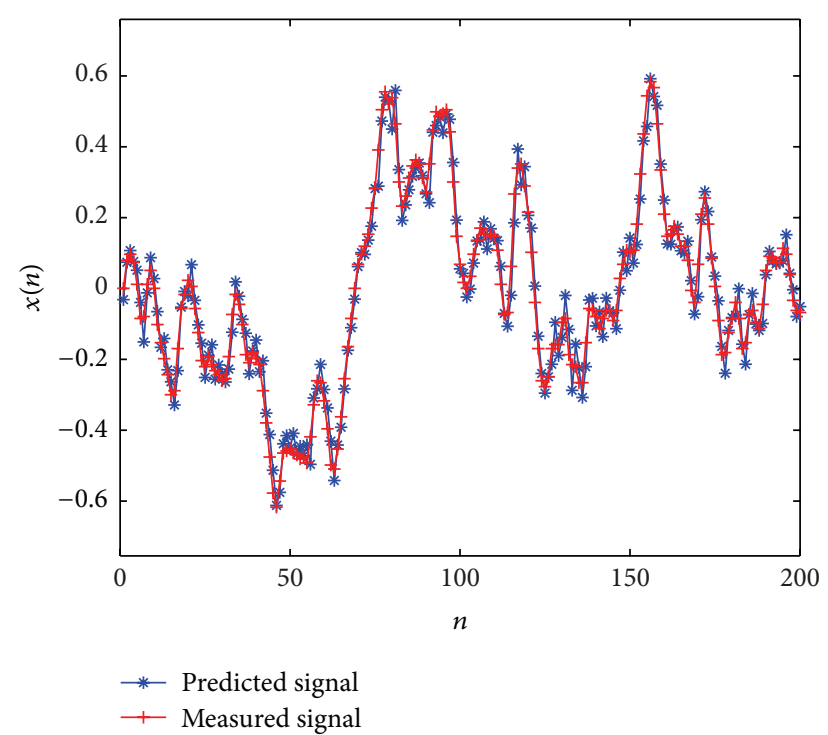

FIGURE 2: Measured signal and predicted signal for the first type of real ship radiated noise (200 points).

comparison, only the first 200 points of the waveform are drawn.

In the same way, optimal individual encoding for the second type of underwater acoustic signal is given by

$$
\left[\begin{array}{llllllll}
1 & 0 & 0 & 0 & 1 & 1 & 0 & 0 \\
0 & 1 & 0 & 0 & 1 & 0 & 0 & 1 \\
0 & 0 & 1 & 0 & 0 & 1 & 1 & 0 \\
0 & 0 & 0 & 1 & 0 & 0 & 2 & 1 \\
0 & 0 & 0 & 0 & 0 & 0 & 0 & 1
\end{array}\right] .
$$

These coefficients are, respectively, $1.8801,-1.6157,0.7905$, $-0.1650,0.0931,-0.1155,-0.1167$, and 0.0694 .

The mathematical expression of the second type is given by the nonlinear functional relation

$$
\begin{aligned}
x(n)= & 1.8801 x(n-1)-1.6157 x(n-2) \\
& +0.7905 x(n-3)-0.165 x(n-4) \\
& +0.0931 x(n-1) x(n-2) \\
& -0.1155 x(n-1) x(n-3) \\
& -0.1167 x(n-3) x(n-4)^{2} \\
& +0.0694 x(n-2) x(n-4) x(n-5),
\end{aligned}
$$

where $x(n)$ is the signal amplitude (Figure 3).

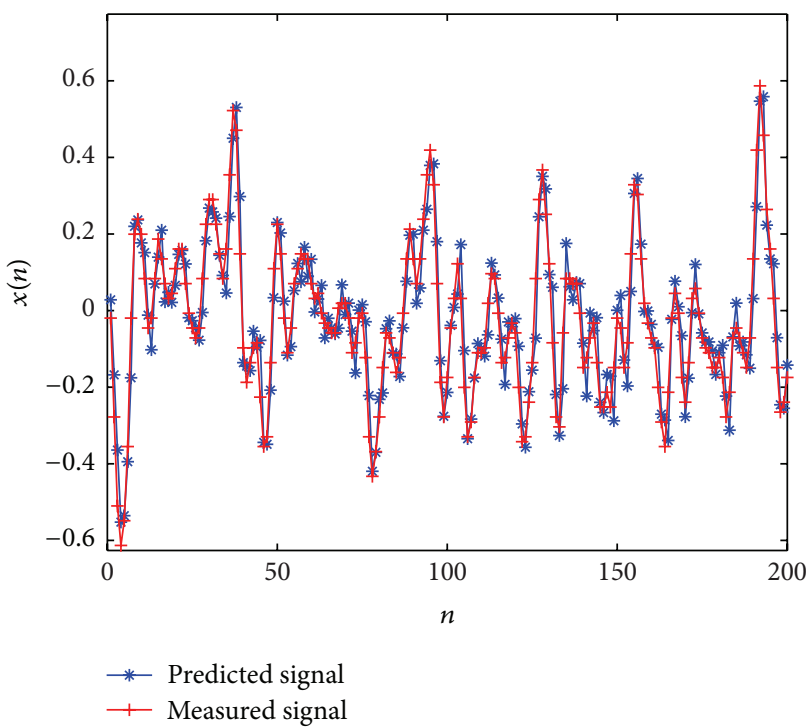

FIgURE 3: Measured signal and predicted signal for the second type of real ship radiated noise (200 points).

In the same way, optimal individual encoding for the third type of underwater acoustic signal is given by

$$
\left[\begin{array}{llllllll}
1 & 0 & 0 & 0 & 3 & 1 & 0 & 0 \\
0 & 1 & 0 & 0 & 0 & 0 & 1 & 0 \\
0 & 0 & 1 & 0 & 0 & 2 & 1 & 1 \\
0 & 0 & 0 & 1 & 0 & 0 & 0 & 0 \\
0 & 0 & 0 & 0 & 0 & 0 & 1 & 2
\end{array}\right] .
$$

These coefficients are, respectively, 1.8034, -1.6079 , $0.9540,-0.2238,-0.1592,0.0138,0.0290$, and -0.1847 .

The mathematical expression of the third type is given by the nonlinear functional relation

$$
\begin{aligned}
x(n)= & 1.8034 x(n-1)-1.6079 x(n-2) \\
& +0.954 x(n-3)-0.2238 x(n-4) \\
& -0.1592 x(n-1)^{3} \\
& +0.0138 x(n-1) x(n-3)^{2} \\
& +0.029 x(n-2) x(n-3) x(n-5) \\
& -0.1847 x(n-3) x(n-5)^{2},
\end{aligned}
$$

where $x(n)$ is the signal amplitude (Figure 4).

In (1), Volterra series have a lot of items. By genetic algorithm, some items are discarded, and others are kept. Then these coefficients are calculated by the least squares method. Lastly, the mathematical expressions of underwater acoustic signal are obtained such as (4), (6), and (8).

\section{Results and Discussions}

In order to quantitatively analyze the effect of underwater acoustic signal predicting modeling method with genetic 


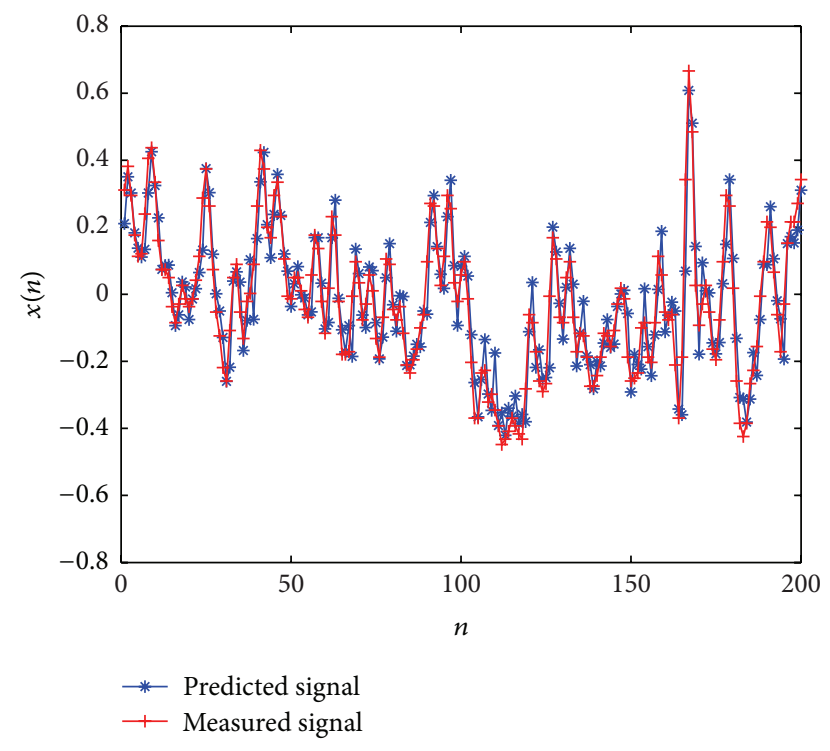

FIGURE 4: Measured signal and predicted signal for the third type of real ship radiated noise (200 points).

TABLE 1: The root mean square error and signal error ratio of underwater acoustic signal prediction.

\begin{tabular}{lcc}
\hline Underwater acoustic signal classification & RMSE & SER \\
\hline The first type of real ship radiated noise & 0.0428 & 14.7 \\
The second type of real ship radiated noise & 0.0660 & 10.1 \\
The third type of real ship radiated noise & 0.0881 & 9.3 \\
\hline
\end{tabular}

algorithm, the root mean square error and the signal error ratio are used.

The root mean square error (RMSE) and the signal error ratio (SER) are, respectively, defined by

$$
\begin{aligned}
\text { RMSE } & =\sqrt{\frac{1}{N} \sum_{n=1}^{N}(\widehat{x}(n)-x(n))^{2},} \\
\text { SER } & =10 \log _{10}\left[\frac{E\left(x(n)^{2}\right)}{E\left((x(n)-\widehat{x}(n))^{2}\right)}\right],
\end{aligned}
$$

where $x(n)$ represents the measured value at $n$ moment and $\widehat{x}(n)$ represents the predicted value at $n$ moment.

The root mean square error and signal error ratio of the prediction are shown in Table 1. It can be seen from Table 1 that modeling method of ship radiated noise with genetic algorithm is feasible. When the ups and downs in the time domain waveform change little, such as the first type of underwater acoustic signal, its modeling effect is best, because the root mean square error and the signal error ratio are 0.0428 and $14.7 \mathrm{~dB}$, respectively.

Three types of underwater acoustic signal are modeled, respectively, by genetic algorithm, and the corresponding mathematical expressions are given, which will play an important role in the subsequent processing of underwater acoustic signal.

\section{Conclusion}

In this paper, we employ recent developments on nonlinear physics and time series prediction to study the physical characteristics of measured underwater acoustic signal. Specifically, we examine the predictability of a sample of ship radiated noise recorded in the South China Sea. To do that, an approach based on genetic algorithms has been employed. By calculating the root mean square error and signal error ratio, the satisfactory results are obtained. The results indicate that the proposed modeling method has higher prediction accuracy and is the foundation for the further processing of underwater acoustic signal such as noise reduction, feature extraction, and classification.

\section{Competing Interests}

The authors declare that they have no competing interests.

\section{References}

[1] Q. Leng, W. H. Song, C. M. Zhao, and G. Q. Wu, "Propagation characteristic of envelope line spectrum of ship radiating noise in shallow water channel," Science China Physics, Mechanics \& Astronomy, vol. 44, no. 2, pp. 134-141, 2014.

[2] X. Zhang, X. Zhang, and L. Lin, "Researches on chaotic phenomena of noises radiated from ships," Acta Acustica, vol. 23, no. 2, pp. 134-140, 1998.

[3] A. G. Song and J. R. Lu, "Nonlinear feature analysis and extraction of ship noise signal based on limit cycle," Acta Acustica, vol. 24, no. 4, pp. 407-415, 1999.

[4] Y. A. Li, X. M. Zhang, and D. M. Xu, "The research on the chaotic characteristics of the signals of the target in the sea," Journal of Detection \& Control, vol. 22, no. 2, pp. 17-21, 2000.

[5] G. H. Li and H. Yang, "A prediction method for underwater acoustic chaotic signal based on RBF neural network," Journal of Software, vol. 9, no. 6, pp. 1581-1586, 2014.

[6] Y. Y. He and H. D. Zhang, "Modeling and prediction of underwater acoustic signal based on PSO and RBF neural network," Computer Engineering, vol. 34, no. 23, pp. 208-213, 2008.

[7] J. N. Zhou, Y. A. Li, and Y. S. Wu, "Prediction of underwater acoustic signals based on neural network," Technical Acoustics, vol. 25, no. 3, pp. 226-229, 2006.

[8] T. Baklacioglu, "Fuel flow-rate modelling of transport aircraft for the climb flight using genetic algorithms," The Aeronautical Journal, vol. 119, no. 1212, pp. 173-183, 2015.

[9] A. Alvarez, C. Harrison, and M. Siderius, "Predicting underwater ocean noise with genetic algorithms," Physics Letters A, vol. 280, no. 4, pp. 215-220, 2001.

[10] J. S. Zhang and X. C. Xiao, "Predicting low-dimensional chaotic time series using volterra adaptive filers," Acta Physica Sinica, vol. 49, no. 3, pp. 407-408, 2000.

[11] Q. F. Meng, Q. Zhang, and W. Y. Mu, "A novel multi-step adaptive prediction method for chaotic time series," Acta Physica Sinica, vol. 55, no. 4, pp. 1666-1671, 2006. 


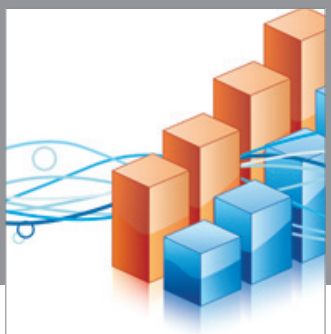

Advances in

Operations Research

vatem alat4

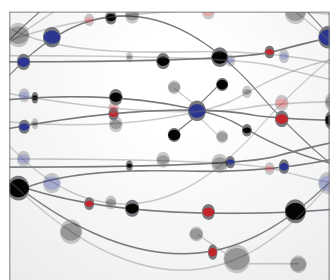

\section{The Scientific} World Journal
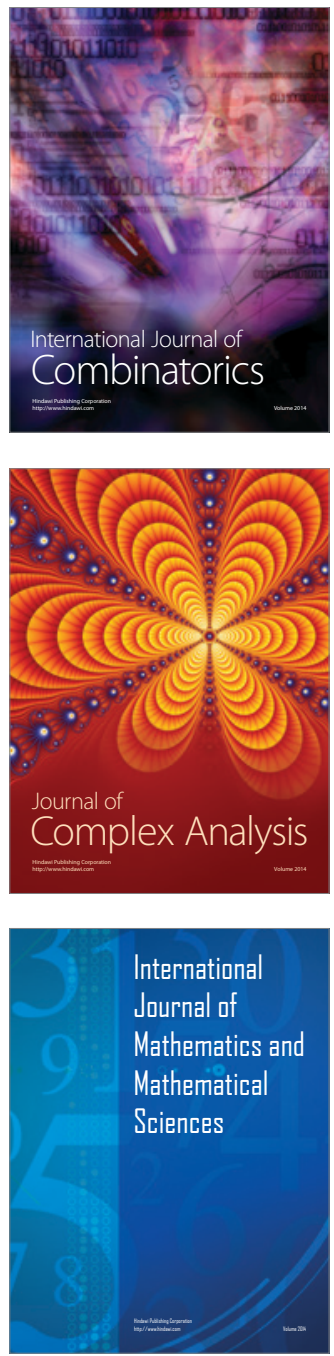
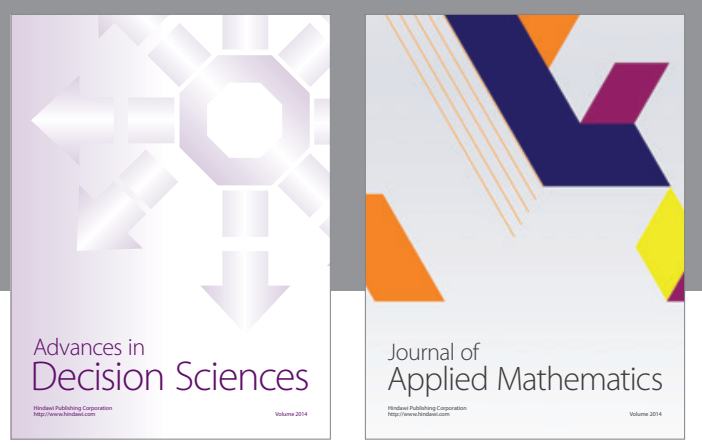

Algebra

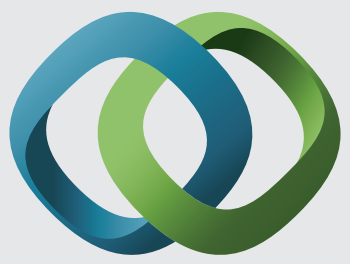

\section{Hindawi}

Submit your manuscripts at

http://www.hindawi.com
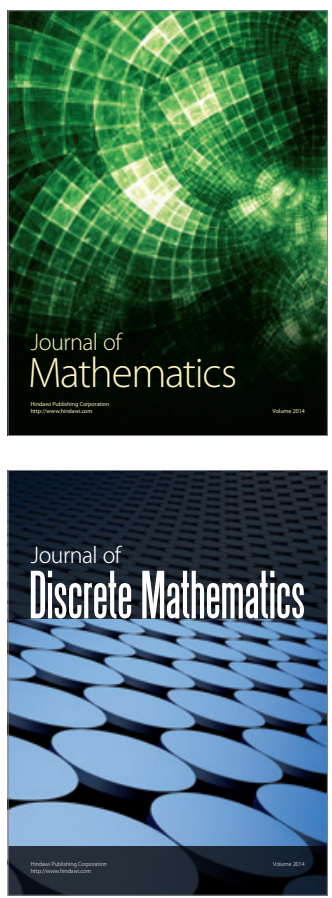

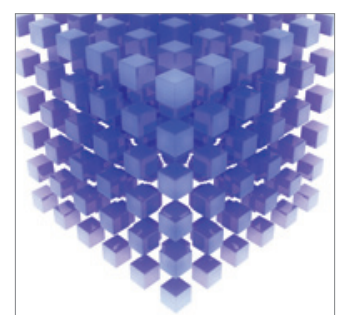

Mathematical Problems in Engineering
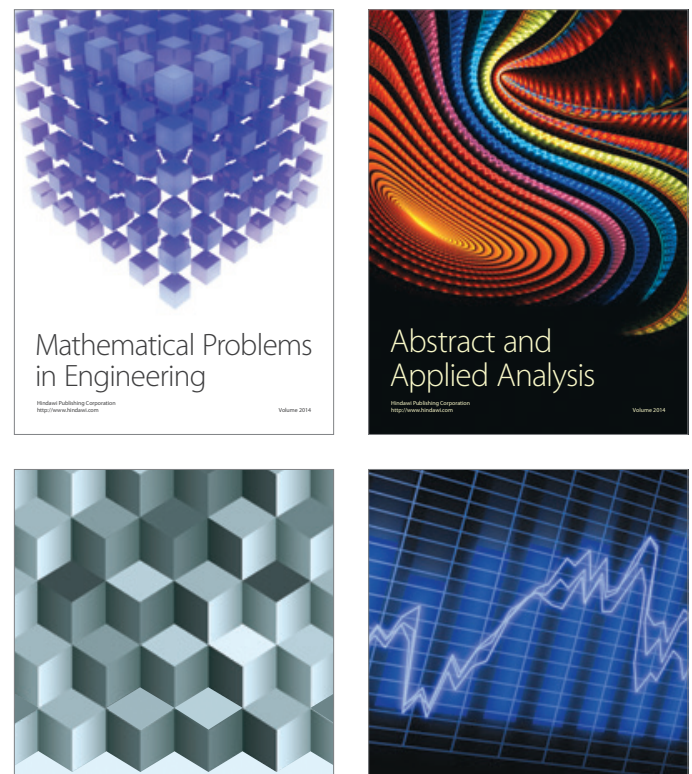

Journal of

Function Spaces

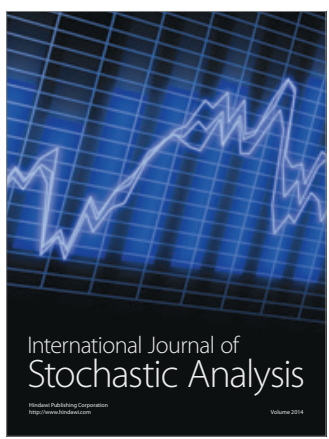

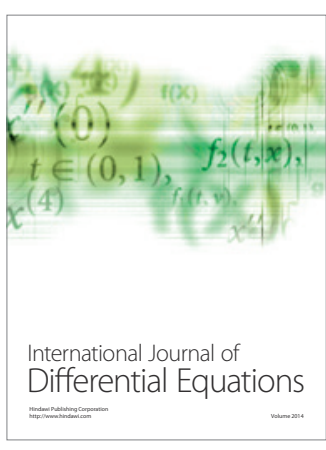
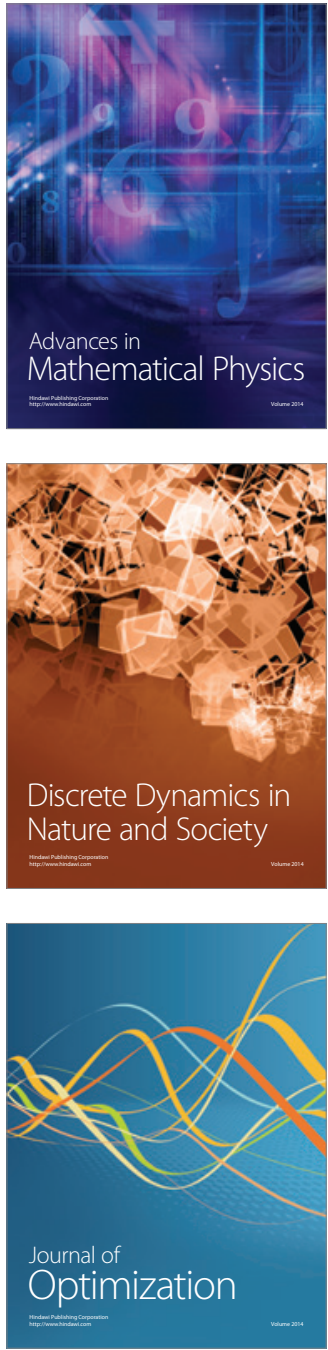